\title{
Hemodynamic Instability and Cardiovascular Events after Traumatic Brain Injury Predict Outcome after Artifact Removal with Deep Belief Network Analysis
}

Hakseung Kim ${ }^{1}, \mathrm{PhD}(\mathrm{C})$, Seung-Bo Lee ${ }^{1}$, MEng, Yunsik Son ${ }^{2}, \mathrm{PhD}$, Marek Czosnyka ${ }^{3}$, $\mathrm{PhD}$, and Dong-Joo $\mathrm{Kim}^{1 *}$, PhD.

${ }^{1}$ Department of Brain and Cognitive Engineering, Korea University, Seoul, South Korea ${ }^{2}$ Department of Computer Engineering, Dongguk University, Seoul, South Korea ${ }^{3}$ Division of Neurosurgery, Department of Clinical Neurosciences, University of Cambridge, Cambridge, UK

*Corresponding author: Dong-Joo Kim, Ph.D., Assistant Professor, Departments of Brain and Cognitive Engineering, Korea University, Anam-dong, Seongbuk-gu, Seoul, South Korea 02841.

Phone: +82-2-3290-5929

Fax: $+82-2-3290-3970$

E-mail: dongjookim@korea.ac.kr.

Conflicts of Interest: The authors declare that there is no conflict of interest.

Source of Funding: This research was supported by a grant from the Korea Health Technology R\&D Project through the Korea Health Industry Development Institute (KHIDI), funded by the Ministry of Health \& Welfare, Republic of Korea (grant number: HI17C1790). 


\section{ABSTRACT}

Background: Hemodynamic instability and cardiovascular events heavily affect the prognosis of traumatic brain injury (TBI). Physiological signals are monitored to detect these events. However, the signals are often riddled with faulty readings, which jeopardize the reliability of the clinical parameters obtained from the signals. A machine learning model for the elimination of artifactual events shows promising results for improving signal quality. However, the actual impact of the improvements on the performance of the clinical parameters after the elimination of the artifacts is not well studied.

Methods: The arterial blood pressure of 99 subjects with TBI was continuously measured for five consecutive days, beginning on the day of admission. The machine learning deep belief network (DBN) was constructed to automatically identify and remove false incidences of hypotension, hypertension, bradycardia, tachycardia, and alterations in cerebral perfusion pressure (CPP).

Results: The prevalences of hypotension and tachycardia were significantly reduced by $47.5 \%$ and $13.1 \%$, respectively, after suppressing false incidents $(\mathrm{p}=0.01)$. Hypotension was particularly effective at predicting outcome favorability and mortality after artifact elimination ( $\mathrm{p}=0.015$ and 0.027 , respectively). In addition, increased CPP was also statistically significant in predicting outcomes $(\mathrm{p}=0.02)$.

Conclusions: The prevalence of false incidents due to signal artifacts can be significantly reduced using machine learning. Some clinical events, such as hypotension and alterations in CPP, gain particularly high predictive capacity for patient outcomes after artifacts are eliminated from physiological signals.

Keywords: Arterial Pressure; Brain Injuries, Traumatic; Machine Learning; Signal Processing, Computer-Assisted 


\section{INTRODUCTION}

Traumatic brain injury (TBI) continues to be one of the leading causes of mortality and morbidity worldwide. ${ }^{1,2}$ Hypotension, hypertension, bradycardia and tachycardia are all critical for the prognosis of TBI; thus, they should be swiftly and accurately recognized. Various physiological signals are measured to detect such clinical events, including the arterial blood pressure (ABP). However, the measured ABP signals are often unstable and riddled with signal artifacts. Clinical parameters derived from artifact-contaminated signals can easily induce erroneous recognition of clinical events; ${ }^{3,4}$ clinicians may either apply excessively aggressive therapy or avoid necessary treatment because of the inaccurately assessed patient's pathological status, resulting in a worse clinical outcome. Thus, suppressing the false incidences of clinical events by improving signal quality is substantially important to better manage the patient's condition. ${ }^{5}$ Given that the continuous monitoring of physiological signals is a hallmark of critical care units, problems due to poor signal quality are the most severe in critical care units, which manage TBI cases.

Many studies have attempted to eliminate signal artifacts measured in critical care units. ${ }^{6,7}$ The algorithms for suppressing false incidences can be categorized into two approaches: statistical approaches, such as time-series analyses, ${ }^{8}$ and artificial intelligence approaches using machine learning techniques. ${ }^{9,10}$ In particular, rapid improvements in machine learning techniques show promising results in the online, real-time detection of signal artifacts..$^{10,11}$

Artificial intelligence also plays a key role in the application of clinical decision systems, which often comprise the algorithms for the computerized derivation of clinical parameters and clinical event detection from continuously measured physiological signals. The quality of physiological signals should be within an acceptable range to ensure the reliability of such systems. ${ }^{12}$ Despite its importance, the literature regarding the impact of 
signal qualities on the performance of derived clinical parameters is insufficient. ${ }^{6}$ In this study, a deep belief network (DBN), which is a machine learning model, was applied to continuously measure ABP signals in TBI patients to detect false incidences of hemodynamic instability (i.e., hypotension and hypertension) and cardiovascular events (i.e., tachycardia and bradycardia). The prevalence of incidences and their predictive capacity on patient outcomes were compared before and after the signal quality control.

\section{MATERIALS AND METHODS}

This study assumed that the false incidence reduction will significantly affect the predictive power of the hemodynamic and cardiovascular parameters for patient outcome, measured by the mortality and six-month Glasgow Outcome Scale. Thereby, the prevalence of hypotension, hypertension, bradycardia and tachycardia were calculated from the continuous recordings of $\mathrm{ABP}$ signals. The change in the mean cerebral perfusion pressure $(\mathrm{CPP}$; calculated as $\mathrm{ABP}$ - intracranial pressure (ICP)) after improving the signal quality was also assessed. The study involved detailed analyses of continuously measured ABP signals (i.e., identification of individual ABP pulses) to allow the automated detection of signal artifacts by the DBN, a machine learning algorithm. A significant portion of the available data set was used to construct, train and validate the DBN. Measuring the efficacy of the constructed DBN by mere accuracy was not deemed feasible, due to the possible bias in data distribution. Such bias could be better addressed via utilizing net prediction rate, defined as (sensitivity + specificity) $/ 2^{13}$; thus, the efficacy of constructed DBN was considered as acceptable if the net prediction rate was satisfactory (higher than 95\%) for classifying signal artifacts. The model was then applied to the total data set to automatically eliminate the signal artifacts. The use of the collected data (ABP and ICP signals), included in an anonymized database for research purposes, was approved by the local ethics committee (29 REC 97/291). 


\section{Data acquisition}

The data from a total of 99 subjects were selected from an anonymized database of TBI patients admitted to the Neurocritical Care Unit of Addenbrooke's Hospital (2004-2010). The following inclusion criteria were applied: 1) patients had severe TBI only; 2) the ABP signal was consecutively recorded with simultaneously measured ICP; and 3) the computersupported recordings started within 24 hours of admission. Considering the variability in the recording duration among the patients, the recordings from a maximum of five consecutive days were analyzed; five days of monitoring for 82 subjects $(82.8 \%)$, four days for four subjects $(4.0 \%)$, three days for six subjects $(6.1 \%)$, two days for six subjects $(6.1 \%)$ and one day for one subject (1.0\%). Accordingly, 9,150 hours of ABP recordings were subjected to the analysis. The proportion of signal artifact was $12.39 \%$ of the total recording time.

The ABP signals were acquired directly from the radial artery (System 8000, S\&W Vickers Ltd., Sidcup, UK; Solar 6000 System, Marquette, USA). The ICP was recorded using an intra-parenchymal probe (Codman ICP MicroSensors, Johnson \& Johnson, Raynham, USA). The subjects underwent intensive care to maintain a CPP between 65 and $75 \mathrm{~mm} \mathrm{Hg}$ and an ICP under $25 \mathrm{~mm} \mathrm{Hg}$ by mild hypocapnia $\left(\mathrm{PaCO}_{2}\right.$, range 4-4.5 kPa), moderate hypothermia, boluses of mannitol ( $200 \mathrm{~mL}$ of $20 \%$ over a period of $2 \mathrm{~min}$ or longer) and hyperventilation induced by thiopentone.

\section{Signal pre-processing}

To derive the heart rate and systolic or diastolic blood pressure from the ABP, the following preprocesses were sequentially performed by two experts: 1) systolic peak detection, 2) pulse onset estimation, and 3) pulse identification. The detailed processes of the systolic peak and pulse onset detection are described elsewhere. ${ }^{14}$ The pulse length and systolic/diastolic 
pressure were calculated from the segmented pulses. The pulses were interpolated using the cubic spline method because the input layer of the DBN receives inputs with a fixed number $(1,024)$ of features. Finally, the pulses were normalized for a probabilistic representation; the normalized pulses were used as the inputs of the DBN for automated artifact elimination.

Determining the proper sizes of training and validation data is an important and delicate matter. Ideally, the sizes should be determined as sufficiently large to construct an effective algorithm (i.e., DBN) yet also sufficiently small to avoid the excessive use of computational power, and in the case of this study, to minimize the burden of the experts who conducted and oversaw the labeling of ABP pulses. Thus, in determining the size of the training data set for DBN, this study started with small-sized training data and then gradually increased the size of the data until satisfactory results were achieved. The target net prediction rate of the DBN for detecting signal artifacts was set at $95 \%$, which was achieved using a randomly selected $30 \%(n=30)$ of the total subjects; $20 \%$ of those $(n=6)$ were used to construct the DBN, and an independent set of data from the rest $(80 \%, \mathrm{n}=24)$ was used for validation of the DBN. This involved the binary labeling of 465,635 ABP pulse waveforms from 6 subjects, and 2,493,095 pulse waveforms from 24 subjects.

The DBN has 6 different layers for processing the input data: 1) the visual layer, which has 1,024 units representing the features of ABP pulses; 2) 4 hidden layers that comprise 128, 50, 10 and 10 units; and finally, 3) an output layer with 2 units, representing the normality of the input data (Figure A2, Supplemental Digital Content 1). The learning rate, momentum and weight decay parameters, which are known to be suitable for classifying the signal data, were set at $0.1,0.9$, and 0.001 , respectively. ${ }^{15,16}$ With the model constructed via training dataset from 6 patients and test dataset from 24 patients, the DBN achieved a sensitivity of $96.3 \%$ and a specificity of $95.4 \%$ for classifying normal and artifactual pulses. Further details on the DBN utilized in this study can be found in the Supplemental Digital 
Content 1.

\section{Criteria for clinical event detection}

Four clinical events (i.e., hypotension, hypertension, bradycardia and tachycardia) were identified from the ABP signals. Systolic pressure $<90 \mathrm{~mm} \mathrm{Hg}$ was defined as hypotension; because all the subjects suffered from severe TBI, systolic pressure $<110 \mathrm{~mm} \mathrm{Hg}$ was also defined as revised hypotension, as per the instructions from the Brain Trauma Foundation. ${ }^{17}$ Systolic pressure $>140 \mathrm{~mm} \mathrm{Hg}$ or diastolic pressure $>90 \mathrm{~mm} \mathrm{Hg}$ was defined as hypertension. The algorithm would detect hypotensive or hypertensive incidences if the duration of such events exceeded 15 seconds. ${ }^{18}$ As per the ANSI/AAMI EC13 cardiac monitor standards, $\mathrm{bpm}<40$ was defined as extreme bradycardia, whereas bpm $>140$ was defined as extreme tachycardia. The algorithm would identify the incidence of bradycardia if extreme bradycardia exceeds 5 seconds or extreme tachycardia continues for $>6$ seconds.

\section{Statistical analyses}

The median value or the interquartile range of every parameter was calculated. The MannWhitney U test was used to compare the parameter values according to the patient outcomes and artifact elimination. The diagnostic odds ratio (DOC) $)^{19}$ in the maximum of Youden's index ${ }^{20}$ was derived from the parameters of $\mathrm{p}<0.05$, which resulted from the Mann-Whitney $\mathrm{U}$ test and assessed the prediction of performance. All statistical analyses were performed using SPSS statistical software version 23 (SPSS IBM Corp, Chicago, USA), and the standard of statistical significance used was set at $p=0.05$.

\section{RESULTS}

\section{Observations}


The mean age of the subjects was 35.8 years (range, 16-74 years), and 71 subjects were male (71.7\%). The mortality rate was $28.3 \%$, and $45.5 \%(n=45)$ of the subjects showed a favorable outcome (Glasgow Outcome Scale $=4-5$ ). The median Glasgow Coma Scale at admission was 6 (range $=3-15)$.

Typical artifact-related false incidences of hypertension are shown in Fig. 1A. The artifacts led to a rapid increase in ABP, reaching approximately $200 \mathrm{~mm} \mathrm{Hg}$. The artifacts caused by the transducer flushing can demonstrate an episode of false hypotension (Fig. 1B). Distortions in the ABP signals can result in false arrhythmias: false tachycardia can originate from significant noise in the ABP signal with extremely shortened pulse lengths (Fig. 1C), whereas false bradycardia may occur due to a few stretched pulses (Fig. 1D).

\section{Prevalence of clinical events after suppressing false incidents}

The prevalence of hypotension, hypertension, bradycardia and tachycardia was evaluated based on the count, duration time and proportion across the entire five-day recordings. Although all hemodynamic instability and cardiovascular events were reduced, only the decreased prevalence of hypotension and tachycardia showed statistical significance $(p<$ $0.05)$.

When assessing the prevalence by count, the bradycardia was the most frequent incidence (Fig. 2A). However, the duration and proportions of hypertensive events exceeded those of bradycardia (Fig. 2B and C). This difference between the count-based and durationbased prevalence was induced by the variation in the duration criteria for detecting each event (over 151.5 seconds for hypertension and 5 seconds for bradycardia). In contrast to the incidence of hypertension, that of hypotension was relatively uncommon (mean count $=2.98$, duration time $=217.3$ seconds, proportion $=0.07 \%$ )

Although all the subjects appeared to have hemodynamic instability and 
cardiovascular events from the raw signals, the incidences among the subjects were decreased by improving the signal quality (Fig. 2D). Specifically, the number of patients with hypotensive events was significantly decreased by $47.5 \% ; 52$ subjects had a strictly artifactinduced false incidence of hypotension. The numbers of subjects with one or more incidences of redefined hypotension and tachycardia were reduced by $12.5 \%$ and $13.1 \%$, respectively. In contrast, the incidences of hypertension and bradycardia decreased only slightly (1\%).

The prevalence of hypotension and tachycardia were significantly reduced $(p=0.01)$ after suppressing the false incidences. The incidences of hypertension and bradycardia were also decreased, but this decrease did not reach statistical significance.

The statistical analyses indicated that only the incidence of hypotension was significant in predicting the outcome favorability and mortality; other hemodynamic instability and cardiovascular events were not predictive of patient outcomes. The prevalence of hypotension, including the count, duration and proportion, showed significant prognostic capacity (Table 1). Overall, the hypotensive incidence gained significance in discriminating the patient outcome after suppressing false incidences. The conventional threshold of systolic pressure $=90 \mathrm{~mm} \mathrm{Hg}$ showed strength in predicting mortality, whereas the redefined threshold was significant in predicting outcome favorability. For both thresholds, the duration of incidence exhibited the best predictive power.

The mean CPP values were significantly underestimated before artifact elimination, and this underestimation caused a detrimental impact on the prognostic value of CPP. The mean CPP values of the favorable and unfavorable outcomes were 75.2 and $71.7 \mathrm{~mm} \mathrm{Hg}$, respectively, when calculated using raw $\mathrm{ABP}$ and ICP signals (Fig. 3). Consequently, the discriminating ability of the CPP did not show statistical significance $(\mathrm{p}=0.07)$. After artifact elimination, the CPP was slightly increased in patients with both favorable and unfavorable outcomes (76.8 and $73.1 \mathrm{~mm} \mathrm{Hg}$, respectively) and showed a significant difference between 
the favorable and unfavorable outcomes $(p=0.02)$.

\section{DISCUSSION}

Continuous monitoring of physiological signals enables the recognition of trends, dynamics and other important characteristics of a patient's pathologic prognosis. Artifact-free signals may enhance the reliability and accuracy of pathophysiologic event detection as well as patient outcomes. ${ }^{6}$ In this study, a machine learning model (i.e., the DBN) was proposed to substantially minimize artifacts from ABP signals. Consequently, this effectively reduces the degree of false incidences of hypotension, hypertension, bradycardia and tachycardia. The results showed that the prevalence of the two cardiovascular incidents were reduced and was not predictive of the patient outcome, regardless of the signal quality.

\section{Prevalence of hemodynamic instability in predicting outcomes}

Hypertension after TBI can increase the blood flow and consequently increase the intracranial blood volume, which can induce vasogenic cerebral edema and intracranial hypertension..$^{21,22}$ Thus, the prevention of prolonged hypertension is one of the important therapeutic goals in TBI. ${ }^{23}$ Indeed, the prevalence of hypertension exhibited predictive power for mortality. Nonetheless, the hypotensive incidence showed the most powerful capacity in predicting a patient's outcome.

Hypotension is a frequent phenomenon in TBI and is associated with a worse outcome and a mortality of up to $65 \% .{ }^{24}$ Hypotension can decrease cerebral perfusion and oxygenation, which could lead to disruption of ionic homeostasis and the development of an intra- or extracellular osmotic gradient, which results in cytotoxic and vasogenic edema. Swollen parenchymal cells might begin to compress capillaries, hence aggravating symptoms by further decreasing the cerebral blood flow. ${ }^{25-27}$ Thus, the 'vicious cycle' in TBI, which is 
critical to secondary injuries that affect the outcome, is more prone to develop under hypotension.

Hypotension has traditionally been defined as systolic pressure $<90 \mathrm{~mm} \mathrm{Hg}$; however, recent studies have suggested that a systolic pressure of $110 \mathrm{~mm} \mathrm{Hg}$ should be the threshold for hypotension in severe TBI patients. ${ }^{28,29}$ The studies concluded that hypotension, redefined as a systolic pressure $<110 \mathrm{~mm} \mathrm{Hg}$, is highly associated with mortality. However, the findings of this study suggest otherwise; the redefined hypotension was significantly associated with higher morbidity (Table 1). This contradiction may have arisen by chance such that the effects of signal quality were not considered in prior studies. Our findings suggest that the conventional threshold of hypotension (systolic pressure $=90 \mathrm{~mm} \mathrm{Hg}$ ) has better predictive power for mortality than the redefined threshold (110 $\mathrm{mm} \mathrm{Hg})$ after eliminating false incidences of hypotension.

After suppressing false incidences, hypotension showed the lowest incidence, which can be explained by the fact that the subjects with TBI underwent intensive management to maintain the CPP level above $65-70 \mathrm{~mm} \mathrm{Hg}$ and to avoid systemic hypotension by fluid loading and vasoactive drugs, such as dopamine $\left(2-15 \mu \mathrm{g} \cdot \mathrm{kg}^{-1} \cdot \mathrm{min}^{-1}\right)$ or norepinephrine $(0.5$ $\left.\mu \mathrm{g} \cdot \mathrm{kg}^{-1} \cdot \mathrm{min}^{-1}\right)$. Despite such management, the hypotensive events still showed a significant association with a worse outcome. Moreover, the predictive power of the hypotension on patient outcome was obscured by the overwhelming number of artifacts. These results strongly suggest that the quality of the signal can affect the clinical decision and alter the predictive capacity of clinical events and derived parameters.

Although not directly associated with hypoxia, as hypotension is, the lowered CPP can also be responsible for the progression of secondary ischemic injury. CPP lower than 70 $\mathrm{mm} \mathrm{Hg}$ is known to be associated with cerebral hypoxia, and although CPP is maintained at a higher level than the threshold, hypoxia may still occur. ${ }^{30}$ Although the incidence of 
hypotension is typically temporal, the decreased CPP can continuously affect the brain oxygenation, whether the underlying cause is increased ICP or decreased ABP. In this sense, the monitoring of CPP would be beneficial in avoiding sustained hypoxic-ischemic damage in severe TBI.

\section{Technical implications}

The classifier for true and false incidences was chosen as the DBN. A recent study utilized neurophysiological signals, namely, electroencephalography (EEG), and compared the performance of the DBN to other classifiers such as the decision tree, support vector machines, and k-nearest neighbors. ${ }^{31}$ The study concluded that DBN outperformed other classifiers. Compared with EEG signals, the ABP is a significantly stable and morphologically simple signal, thus enabling the classification of the artifacts with relative ease using the DBN.

One major advantage of the DBN is its low dependency on data dimensionality. Although not tested in this study, the independence on input data dimensionality can be a significant advantage in patient monitoring at critical care units, which often involves 4 or more types of physiological signals. Considering the trends toward multimodal monitoring in TBI, the use of the DBN over other types of machine learning-based classifiers seems feasible for accurate clinical event detection. Another advantage of DBN is its rapidness of execution, ${ }^{31,32}$ due to its the relatively simple structure compared to that of other deep learning algorithms. Training the DBN may require computational complexity, yet testing the use of the DBN is extremely fast. ${ }^{33}$ With its rapid response to input data, the DBN can detect various types of artifacts in ABP signals in the online environment. Provided with automated and fast-pulse segmentation preprocess, the communication delay between the DBN and realtime signal is nearly absent. In this study, a time buffer of 10 seconds was sufficient to 
conduct real-time analysis.

\section{Limitations and suggestions}

The detection of arrhythmias (i.e., bradycardia and tachycardia) utilized the pulse onset in the ABP signal, not the QRS complex in the electrocardiogram signals. Thus, the situation detected from clean electrocardiogram signals was not considered with the simultaneously measured ABP artifact. Further study is required to increase the reliability of detecting arrhythmia incidences by applying deep learning to ABP and the electrocardiogram signal concurrently. Future studies could also attempt to identify and differentiate various types of signal artifacts. This study encourages the use of machine learning in clinical environments, particularly in (neuro)intensive care units, for analyzing vast amounts of continuously measured physiological signals.

\section{Concluding remarks}

The prevalence of both hemodynamic instability and cardiovascular events becomes significantly reduced after improving the signal quality. The incidents of hemodynamic instability (i.e., hypertension and hypotension) gained significant predictive capacity for patient mortality. The prevalence of hypotension was particularly effective in predicting a favorable outcome. The use of the DBN upon ABP allows for rapid artifact elimination and may contribute to clinical decision, patient care, and the effectiveness of clinical parameters.

\footnotetext{
ABBREVIATIONS

ABP: arterial blood pressure; CPP: cerebral perfusion pressure; DBN: deep belief network; DOC: diagnostic odds ratio; EEG: electroencephalography; ICP: intracranial pressure; TBI: traumatic brain injury.
} 


\section{ACKNOWLEDGMENTS}

Many thanks to all the staff of the Neuro Critical Care Unit in Addenbrooke's Hospital, Cambridge UK for their support and professional help with computer pressure recording in TBI patients. 


\section{REFERENCES}

1. Hyder AA, Wunderlich CA, Puvanachandra P, et al. The impact of traumatic brai n injuries: a global perspective. NeuroRehabilitation 2007;22:341-53.

2. Rusnak M. Traumatic brain injury: giving voice to a silent epidemic. Nat Rev Ne urol 2013;9:186-87.

3. McGhee $\mathrm{BH}$, Bridges EJ. Monitoring arterial blood pressure: what you may not $\mathrm{k}$ now. Crit Care Nurse 2002;22:60-79.

4. Knight BP, Pelosi F, Michaud GF, et al. Physician interpretation of electrocardiog raphic artifact that mimics ventricular tachycardia. Am J Med 2001;110:335-38.

5. Rangayyan RM, Reddy NP. Biomedical signal analysis: a case-study approach. $A$ nn Biomed Eng 2002;30:983.

6. Nizami S, Green JR, McGregor C. Implementation of artifact detection in critical care: a methodological review. IEEE Rev Biomed Eng 2013;6:127-42.

7. Imhoff M, Kuhls S. Alarm algorithms in critical care monitoring. Anesth Analg 2 006;102:1525-37.

8. Imhoff M, Bauer M, Gather U, et al. Statistical pattern detection in univariate tim e series of intensive care on-line monitoring data. Intensive Care Med 1998;24:13 05-14.

9. Li Q, Clifford GD. Signal quality and data fusion for false alarm reduction in the intensive care unit. $J$ Electrocardiol 2012;45:596-603.

10. Zhang Y, Szolovits P. Patient-specific learning in real time for adaptive monitorin $\mathrm{g}$ in critical care. $J$ Biomed Inform 2008;41:452-60.

11. Chen L, Dubrawski A, Wang D, et al. Using supervised machine learning to clas sify real alerts and artifact in online multi-signal vital sign monitoring data. Crit Care Med 2016;44:e456-e63. 
12. McShea M, Holl R, Badawi O, et al. The eICU research institute - a collaboratio $\mathrm{n}$ between industry, health-care providers, and academia. IEEE Eng Med Biol Ma g 2010;29:18-25.

13. Fei M, Irwin GW, Ma S. Life System Modeling and Simulation: International Co nference on Life System Modeling, and Simulation, LSMS 2007, Shanghai, China , September 14-17, 2007 Proceedings. Berlin Heidelberg: Springer, 2007.

14. Lee HJ, Jeong EJ, Kim H, et al. Morphological feature extraction from a continu ous intracranial pressure pulse via a peak clustering algorithm. IEEE Trans Biome d Eng 2016;63:2169-76.

15. Hinton G. A practical guide to training restricted Boltzmann machines. Momentu m 2010;9:926.

16. Bengio Y. Deep learning of representations for unsupervised and transfer learning . Unsuperv Transf Learn Chall Mach Learn 2012;7:19.

17. Carney N, Totten AM, O'Reilly C, et al. Guidelines for the management of sever e traumatic brain injury, fourth edition. Neurosurgery 2016;81:E2.

18. Chambrin MC. Alarms in the intensive care unit: how can the number of false al arms be reduced? Crit Care 2001;5:184-8.

19. Glas AS, Lijmer JG, Prins $\mathrm{MH}$, et al. The diagnostic odds ratio: a single indicato r of test performance. J Clin Epidemiol 2003;56:1129-35.

20. Zhou X-H, McClish DK, Obuchowski NA. Statistical methods in diagnostic medi cine. Hoboken: John Wiley \& Sons, 2009.

21. Shiozaki T. Hypertension and head injury. Curr Hypertens Rep 2005;7:450-3.

22. Haddad SH, Arabi YM. Critical care management of severe traumatic brain injury in adults. Scand J Trauma, Resusc Emerg Med 2012;20:12.

23. Sellmann T, Miersch D, Kienbaum P, et al. The impact of arterial hypertension o 
n polytrauma and traumatic brain injury. Dtsch Arztebl Int 2012;109:849-56.

24. Chesnut RM, Marshall SB, Piek J, et al. Early and late systemic hypotension as a frequent and fundamental source of cerebral ischemia following severe brain inju ry in the Traumatic Coma Data Bank. In: Unterberg AW, Schneider G-H, Lanksc $\mathrm{h}$ WR, eds. Monitoring of Cerebral Blood Flow and Metabolism in Intensive Car e. Vienna: Springer, 1993:121-25.

25. Claassen J, Carhuapoma JR, Kreiter KT, et al. Global cerebral edema after subara chnoid hemorrhage: frequency, predictors, and impact on outcome. Stroke 2002;33 $: 1225-32$.

26. Aldrich EF, Eisenberg HM, Saydjari C, et al. Diffuse brain swelling in severely $\mathrm{h}$ ead-injured children. A report from the NIH Traumatic Coma Data Bank. J Neur osurg 1992;76:450-4.

27. Adelson PD, Clyde B, Kochanek PM, et al. Cerebrovascular response in infants a nd young children following severe traumatic brain injury: a preliminary report. $P$ ediatr Neurosurg 1997;26:200-7.

28. Berry C, Ley EJ, Bukur M, et al. Redefining hypotension in traumatic brain injur y. Injury 2012;43:1833-7.

29. Hasler RM, Nuesch E, Juni P, et al. Systolic blood pressure below $110 \mathrm{~mm} \mathrm{Hg} \mathrm{i}$ $\mathrm{s}$ associated with increased mortality in blunt major trauma patients: multicentre $\mathrm{c}$ ohort study. Resuscitation 2011;82:1202-7.

30. Schmidt JM, Ko SB, Helbok R, et al. Cerebral perfusion pressure thresholds for brain tissue hypoxia and metabolic crisis after poor-grade subarachnoid hemorrhag e. Stroke 2011;42:1351-6.

31. Wulsin DF, Gupta JR, Mani R, et al. Modeling EEG waveforms with semi-super vised deep belief nets: fast classification and anomaly measurement. $J$ Neural Eng 
2011;8:036015.

32. Dahl GE, Yu D, Deng L, et al. Context-dependent pre-trained deep neural networ ks for large-vocabulary speech recognition. IEEE Trans Audio Speech Lang Proce ss 2012;20:30-42.

33. Bernard K, Tarabalka Y, Angulo J, et al. Spectral-spatial classification of hypersp ectral data based on a stochastic minimum spanning forest approach. IEEE Trans Image Process 2012;21:2008-21. 

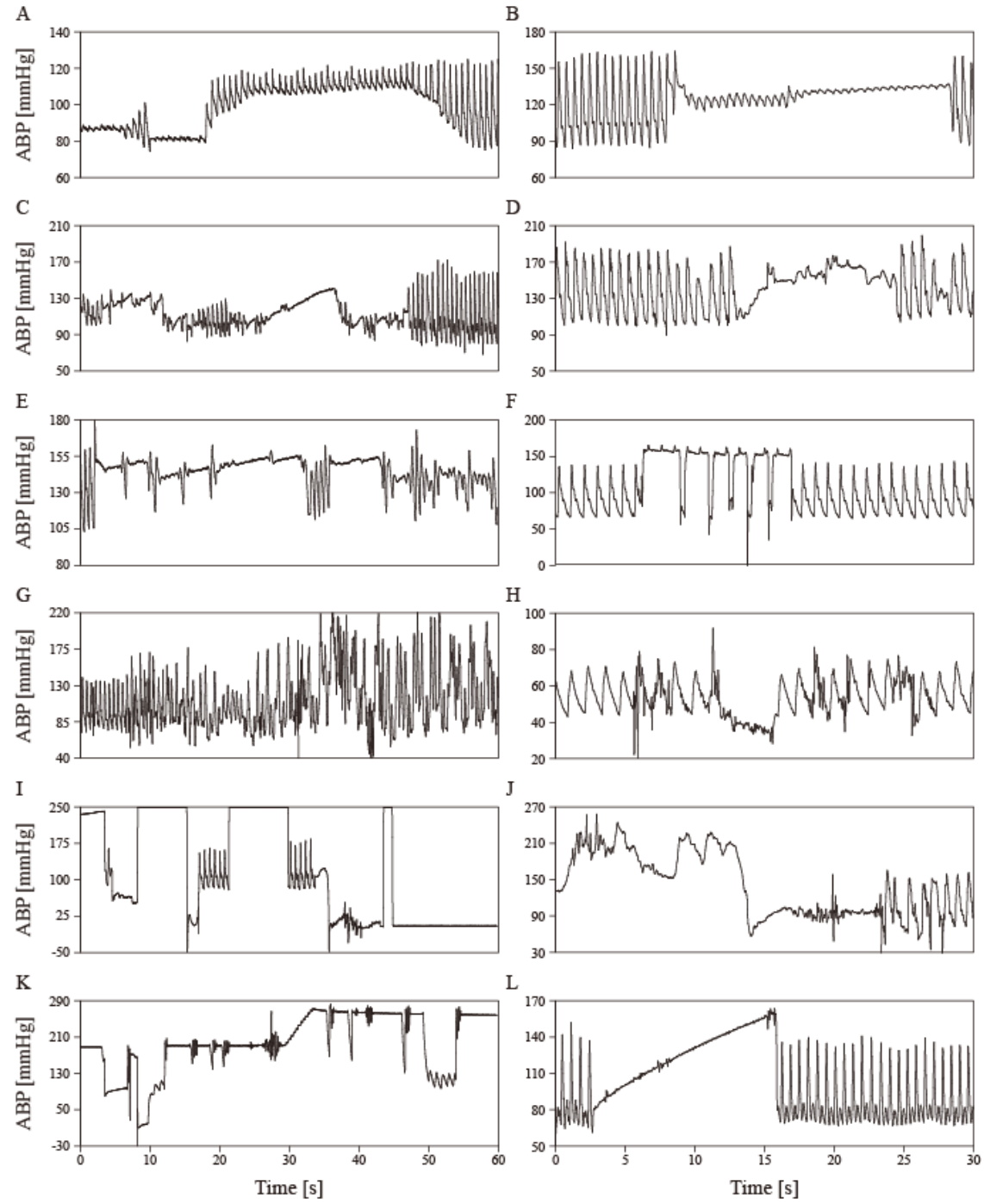

FIGURE 1. A binary classification model based on the DBN is applied to ABP recordings to differentiate artifactual signals. Experimental results (see Supplemental Digital Content 1) and observations (Fig. 1) show that the model classified normal and artifactual signals effectively in various cases, with an accuracy above 96\%. Examples of reduced pulse pressure artifacts are given in Figs. 1A and 1B. This type of artifact causes a misinterpretation of mean pressure, owing to damping caused by a thrombus in the arterial line. Artifacts resulting from motion or patient movement are shown in Figs. 1C-H. The signal corrupted by these artifacts was falsely considered bradycardia or tachycardia. Figure 1I-L describes typical artifact cases caused by transducer flushing or patient movement. These types of artifacts are misinterpreted as hypotension or hypertension. 
A

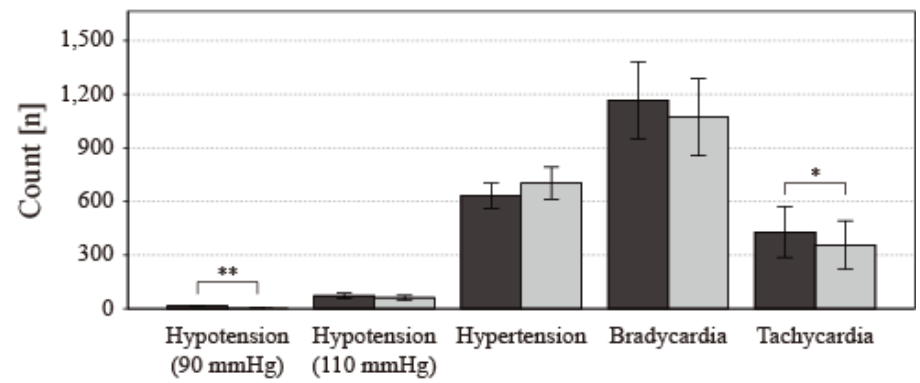

B

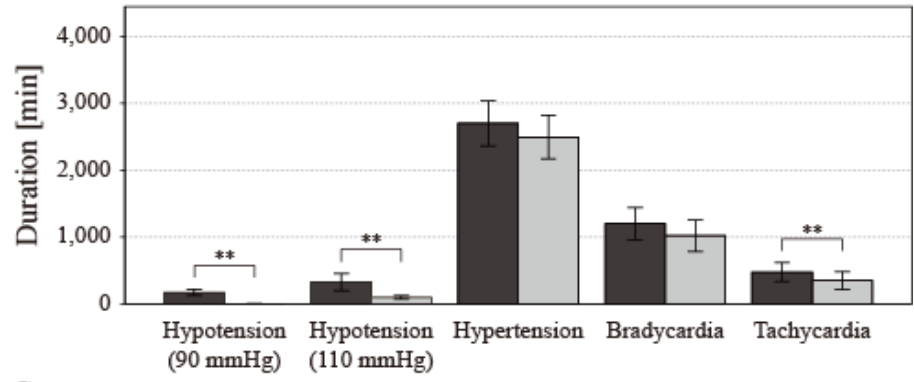

$\mathrm{C}$

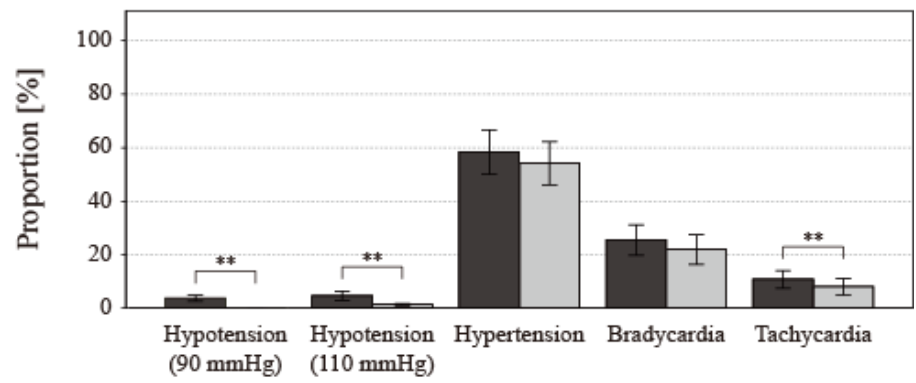

$\mathrm{D}$

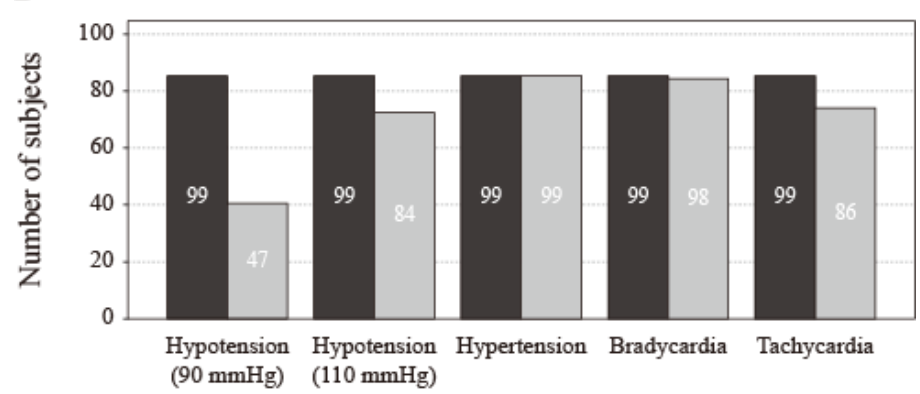

FIGURE 2. Bar graphs showing the incidences of conventional and revised hypotension, hypertension, bradycardia and tachycardia within the five-day recordings, measured as the counts (A), duration time (B) and proportions of the entire recordings (C). The black bars show the mean prevalence detected from the raw signals, whereas the gray bars show the mean prevalence detected from the clean signals. The number of subjects with one or more incidences during the recording times was also compared (D). The stars above the bars indicate statistical significance; one star for $\mathrm{p}<0.05$ and two stars for $\mathrm{p}<0.01$. 


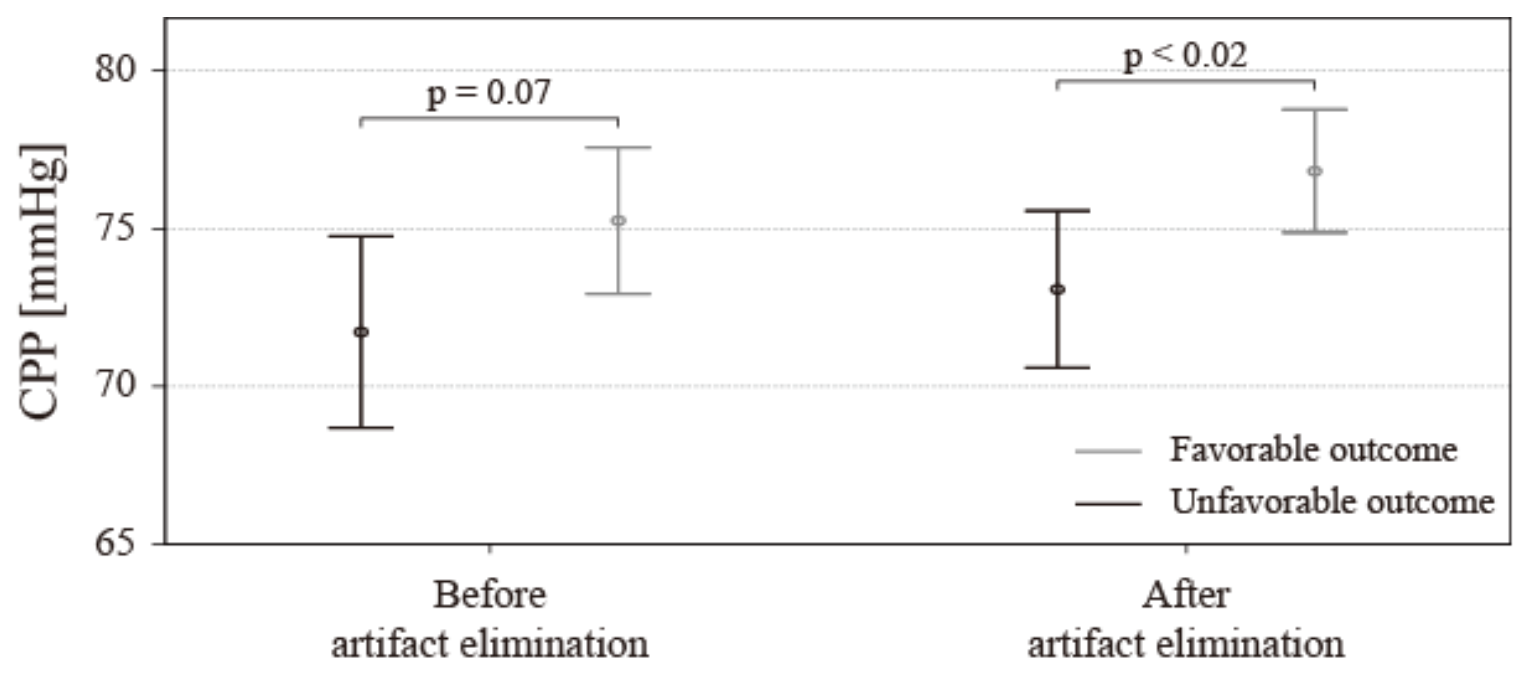

FIGURE 3. Differences in the mean CPP between patients with favorable and unfavorable outcomes before and after artifact elimination. 
TABLE 1. Comparison of hypotensive incidences between the survived and deceased subjects.

\begin{tabular}{|c|c|c|c|c|c|c|c|c|c|}
\hline \multirow[b]{2}{*}{$\begin{array}{c}\text { Artifact } \\
\text { elimination }\end{array}$} & \multirow[b]{2}{*}{$\begin{array}{l}\text { Hypotensive } \\
\text { incidence }\end{array}$} & \multicolumn{2}{|c|}{ Median (IQR) } & \multirow[b]{2}{*}{$\begin{array}{c}\text { OR } \\
(95 \% \mathrm{CI})^{*}\end{array}$} & \multirow[b]{2}{*}{$\begin{array}{c}\mathrm{P}- \\
\text { value }\end{array}$} & \multicolumn{2}{|c|}{ Median (IQR) } & \multirow[b]{2}{*}{$\begin{array}{l}\text { OR }(95 \% \\
\text { CI })^{*}\end{array}$} & \multirow[b]{2}{*}{$\begin{array}{c}\text { P- } \\
\text { value }\end{array}$} \\
\hline & & $\begin{array}{l}\text { Survival } \\
(\mathrm{N}=71)\end{array}$ & $\begin{array}{c}\text { Dead } \\
(\mathrm{N}=28)\end{array}$ & & & $\begin{array}{l}\text { Favourable outcome } \\
\qquad(\mathrm{N}=45)\end{array}$ & $\begin{array}{c}\text { Unfavourable } \\
\text { outcome } \\
(\mathrm{N}=44)\end{array}$ & & \\
\hline \multirow{8}{*}{ 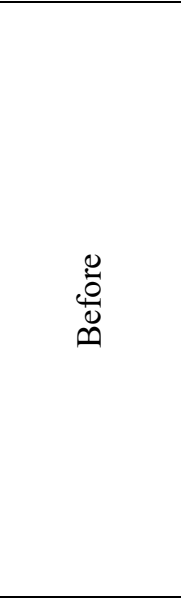 } & Conventional $l^{\dagger}$ & & & & & & & & \\
\hline & Count $[\mathrm{n}]$ & $\begin{array}{c}11 \\
(7-18.25)\end{array}$ & $\begin{array}{c}12 \\
(6.5-18.25)\end{array}$ & & 0.85 & $\begin{array}{c}10.5 \\
(6.25-18)\end{array}$ & $\begin{array}{c}12 \\
(8-19)\end{array}$ & & 0.617 \\
\hline & Time $[\mathrm{min}]$ & $\begin{array}{c}86.82 \\
(15.43-201.62)\end{array}$ & $\begin{array}{c}62.65 \\
(12.14-258.33)\end{array}$ & & 0.80 & $\begin{array}{c}67.75 \\
(13.57-222.08)\end{array}$ & $\begin{array}{c}87.21 \\
(15.04-214.41)\end{array}$ & & 0.597 \\
\hline & Proportion [\%] & $\begin{array}{c}1.67 \\
(0.33-4.37)\end{array}$ & $\begin{array}{c}1.45 \\
(0.08-5.98)\end{array}$ & & 0.86 & $\begin{array}{c}1.26 \\
(0.21-4.41)\end{array}$ & $\begin{array}{c}1.69 \\
(0.3-4.67)\end{array}$ & & 0.726 \\
\hline & Redefined $^{\S}$ & & & & & & & & \\
\hline & Count $[\mathrm{n}]$ & $\begin{array}{c}45 \\
(15-125.75)\end{array}$ & $\begin{array}{c}50 \\
(26.75-72.25)\end{array}$ & & 0.83 & $\begin{array}{c}24.5 \\
(11-78)\end{array}$ & $\begin{array}{c}64 \\
(25.5-100.25)\end{array}$ & & 0.01 \\
\hline & Time [min] & $\begin{array}{c}175.79 \\
(63.07-415.19)\end{array}$ & $\begin{array}{c}153.12 \\
(81.54-313.28)\end{array}$ & & 0.96 & $\begin{array}{c}142.51 \\
(42.63-412.42)\end{array}$ & $\begin{array}{c}182.4 \\
(96.53-355.4)\end{array}$ & & 0.217 \\
\hline & Proportion [\%] & $2.63(1.08-5.82)$ & $\begin{array}{c}2.13 \\
(1.13-6.24) \\
\end{array}$ & & 0.96 & $\begin{array}{c}2.3 \\
(0.63-5.8) \\
\end{array}$ & $\begin{array}{c}2.64 \\
(1.37-6.51) \\
\end{array}$ & & 0.239 \\
\hline \multirow{8}{*}{$\underbrace{\grave{E}}_{\bar{E}}$} & Conventional & & & & & & & & \\
\hline & Count $[\mathrm{n}]$ & $\begin{array}{c}0 \\
(0-2)\end{array}$ & $\begin{array}{l}1.5 \\
(0-7)\end{array}$ & $\begin{array}{c}2.70 \\
(1.08-6.70)\end{array}$ & 0.03 & $\begin{array}{c}0 \\
(0-2)\end{array}$ & $\begin{array}{c}1 \\
(0-7)\end{array}$ & $\begin{array}{c}2.61 \\
(1.14-5.94)\end{array}$ & 0.02 \\
\hline & Time [min] & $\begin{array}{c}0 \\
(0-1.79)\end{array}$ & $\begin{array}{c}1.18 \\
(0-7.5)\end{array}$ & $\begin{array}{c}3.24 \\
(1.30-8.09)\end{array}$ & 0.02 & $\begin{array}{c}0 \\
(0-1.54)\end{array}$ & $\begin{array}{c}0.47 \\
(0-5.76)\end{array}$ & $\begin{array}{c}2.61 \\
(1.14-5.94)\end{array}$ & 0.03 \\
\hline & Proportion [\%] & $\begin{array}{c}0 \\
(0-0.03)\end{array}$ & $\begin{array}{c}0.02 \\
(0-0.12)\end{array}$ & $\begin{array}{c}2.96 \\
(1.20-7.32)\end{array}$ & 0.02 & $\begin{array}{c}0 \\
(0-0.03)\end{array}$ & $\begin{array}{c}0.01 \\
(0-0.1)\end{array}$ & $\begin{array}{c}2.61 \\
(1.14-5.94)\end{array}$ & 0.04 \\
\hline & Redefined $^{\S}$ & & & & & & & & \\
\hline & Count $[\mathrm{n}]$ & $\begin{array}{c}28.5 \\
(5-104.5)\end{array}$ & $\begin{array}{c}40.5 \\
(11-65)\end{array}$ & $\begin{array}{c}2.23 \\
(0.87-5.74)\end{array}$ & 0.66 & $\begin{array}{c}13 \\
(1-70.5)\end{array}$ & $\begin{array}{c}51.5 \\
(11-94)\end{array}$ & $\begin{array}{c}3.95 \\
(1.69-9.19)\end{array}$ & 0.005 \\
\hline & Time $[\mathrm{min}]$ & $\begin{array}{c}30.96 \\
(5.23-117.97)\end{array}$ & $\begin{array}{c}48.9 \\
(17.45-97.09)\end{array}$ & $\begin{array}{c}3.36 \\
(1.27-8.92)\end{array}$ & 0.22 & $\begin{array}{c}12.14 \\
(0.99-56.01)\end{array}$ & $\begin{array}{c}66.99 \\
(13.24-149.18)\end{array}$ & $\begin{array}{c}5.52 \\
(2.31-13.20)\end{array}$ & $\begin{array}{c}< \\
0.001\end{array}$ \\
\hline & Proportion [\%] & $\begin{array}{c}0.43 \\
(0.07-1.64)\end{array}$ & $\begin{array}{c}0.72 \\
(0.27-1.39)\end{array}$ & $\begin{array}{c}3.36 \\
(1.27-8.92)\end{array}$ & 0.24 & $\begin{array}{c}0.19 \\
(0.01-1.17)\end{array}$ & $\begin{array}{c}0.93 \\
(0.2-2.07)\end{array}$ & $\begin{array}{c}5.52 \\
(2.31-13.20)\end{array}$ & 0.003 \\
\hline
\end{tabular}

${ }^{\dagger}$ systolic pressure $<90 \mathrm{~mm} \mathrm{Hg}$; ${ }^{\S}$ systolic pressure $<110 \mathrm{~mm} \mathrm{Hg}$; ${ }^{*}$ odds ratio. Statistical significance (p-value $<0.05$ ) is indicated in bold character. 


\section{Supplemental Digital Content 1}

Deep belief network; performance optimization

To eliminate artifacts from continuously measured arterial blood pressure (ABP) signals, a deep belief network (DBN) model was constructed that can distinguish between normal and artifactual signals by the following sequences: 1) labeling procedure, 2) pulse interpolation and normalization, and 3) model construction (training and testing). In this endeavor, the ABP pulses were first segmented by analyzing basic morphological features of pulse waveforms (e.g., onset, peaks, troughs, and flats $)^{1,2}$.

Among the cohort of patients with traumatic brain injury (TBI), ABP recordings of 30 subjects were randomly selected (mean recording times $=23.7 \pm 3.1$ hours). The continuous ABP signals were segmented into pulses and were labeled normal or artifact by two experts using in-house software developed in C\# (Fig. A1). Consequently, a total of 2,958,739 pulses were binary labeled.

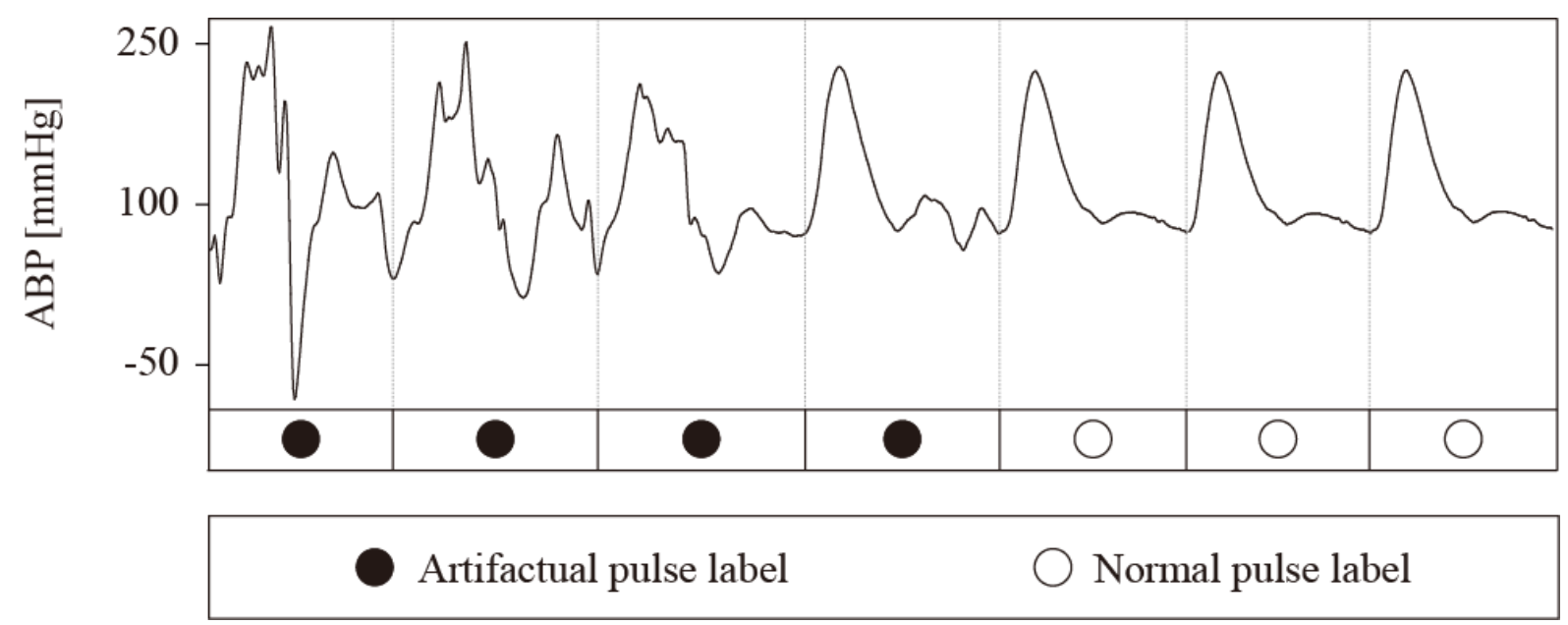

FIGURE A1. Brief description of the labeling segmented ABP pulses

The number of data points in the segmented pulses varies according to the pulse length and sampling frequencies. Because the input layer of the DBN receives inputs with a 
fixed number of features, all pulses should be interpolated to have the same number of data points as the number of units in the input layer. Accordingly, the varying numbers of data points among the pulse segments were adjusted to have exactly the same number of data points $(1,024)$ via the cubic spline interpolation method, which is commonly used for signal interpolation. ${ }^{3}$ Finally, the segmented pulses with constant data points were normalized to the range of $[0,1]$ in dimension for a probabilistic representation according to the normalization function. The normalization was intended to minimize the effects of features other than the morphology of the ABP pulse segments (e.g., trends in ABP pulse) in the learning capacity of the DBN, which would allow better learning of signal morphology for the DBN.

The upper and lower bounds were the maximum and minimum pressure values of each pulse, respectively. By normalizing the pressure value of each data point, the ABP signal could be fed into the input layer of the DBN.

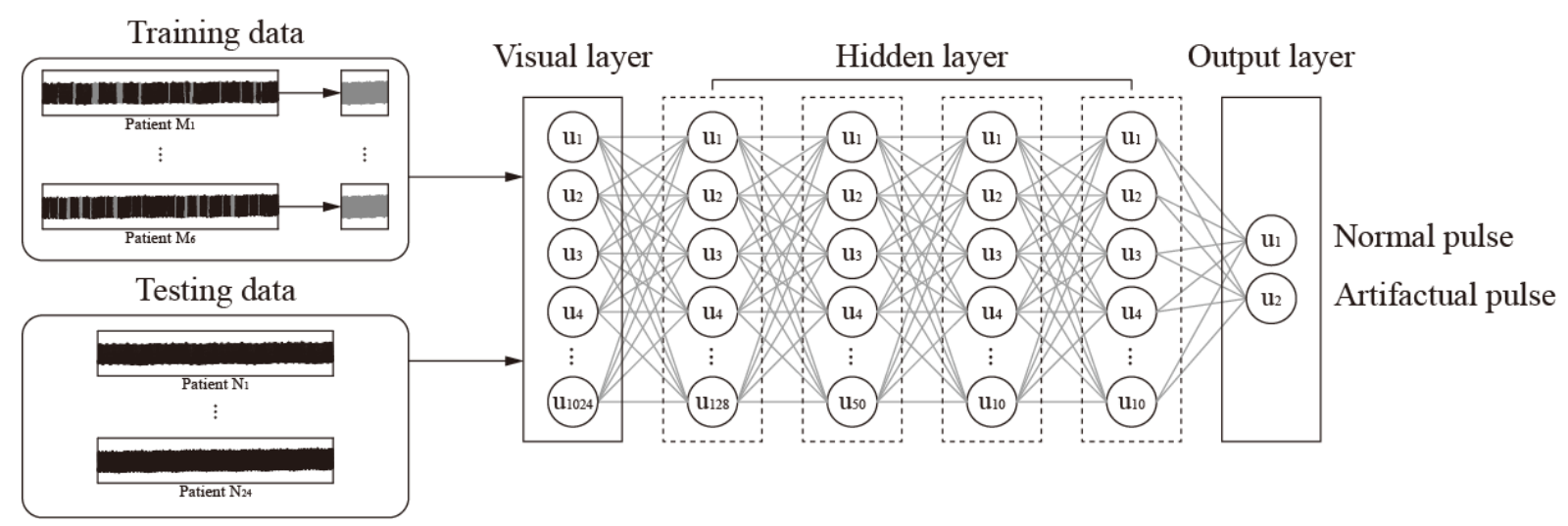

FIGURE A2. DBN construction procedure with training $(n=6)$ and testing data $(n=24)$.

To train the DBN, 6 subjects ( $20 \%$ of 30 subjects) were randomly selected, and $20 \%$ of $\mathrm{ABP}$ pulses were randomly extracted from the recordings of each subject to construct a training data set (Fig. A2). The structure of the DBN comprises 5 hidden layers with 128, 50, 10, 10 and 2 units. The hyper-parameters of the DBN, namely, the learning rate, momentum 
and weight decay, were set as $0.1,0.9$ and 0.001 , respectively, which are values known to be suitable for classifying the signal data. ${ }^{4-6}$ The major components of the DBN, such as the joint distribution and energy function, were the same as those in the conventional DBN models. $^{7-9}$

The number of optimal iterations for training was investigated from 10 to $50,100,500$ and 1,000 times. The performance of the constructed model was analyzed with the remaining ABP recordings of 24 subjects $(80 \%)$, which were clearly distinguished from the training data. The sensitivity (recall), specificity, positive likelihood ratio (LR+), negative LR (LR-), accuracy and net prediction were calculated to assess the classification performances for classifying normal and artifact signals.

A true positive was defined as the proportion of artifact signals that were correctly classified using the DBN model, whereas a true negative was the proportion of normal signals that were correctly classified using the model. In addition, the proportion of artifact signals classified as normal was false negatives, and the proportion of normal signals classified as artifacts was false positives in the same way.

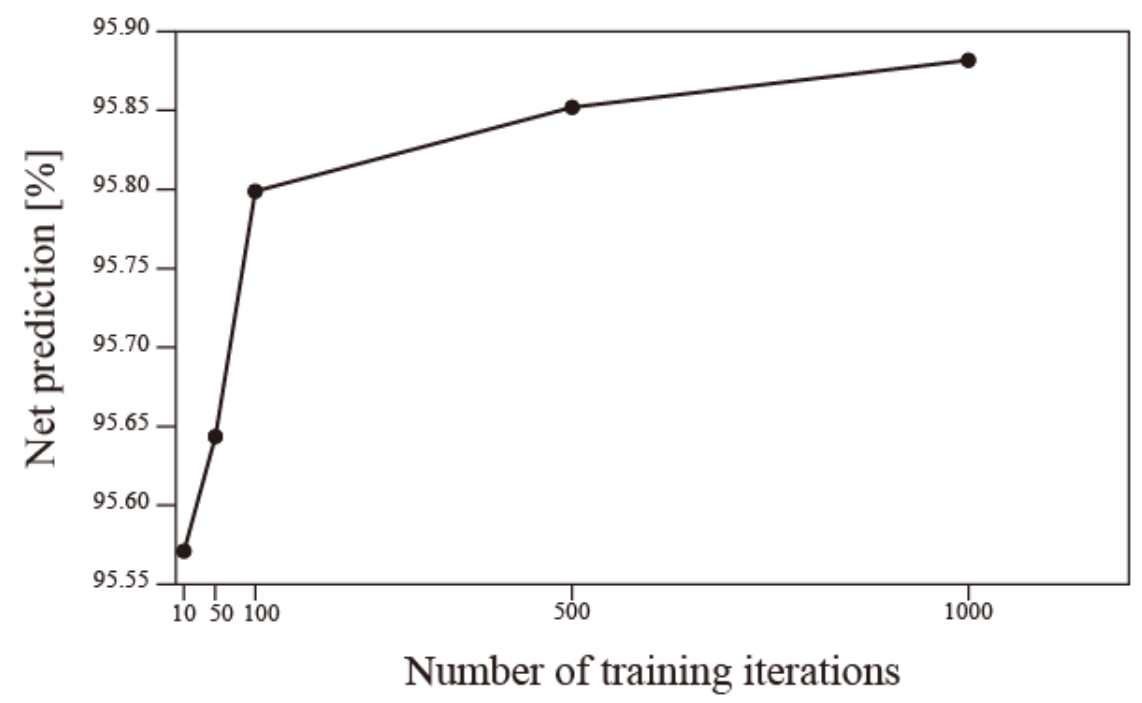

FIGURE A3. Net prediction for artifact detection according to the number of training 
iterations.

The performance of artifact detection according to the number of training iterations increased logarithmically (Fig. A3). In 1,000 training iterations, the networks were judged to be well-learned; therefore, these networks were called optimized networks. The optimized network of the DBN, built with training data set from 6 patients and test dataset from 24 patients, exhibited a sensitivity of $96.3 \%$, a specificity of $95.4 \%$, an LR+ of 21.05 , an LR- of 0.04 , an accuracy of $95.5 \%$ and a net prediction of $95.9 \%$ for classifying normal and artifactual pulses.

\section{REFERENCES}

1. Lee HJ, Jeong EJ, Kim H, et al. Morphological feature extraction from a continu ous intracranial pressure pulse via a peak clustering algorithm. IEEE Trans Biome d Eng 2016;63:2169-76.

2. Li BN, Dong MC, Vai MI. On an automatic delineator for arterial blood pressure waveforms. Biomed Signal Process Control 2010;5:76-81.

3. McKinley S, Levine M. Cubic spline interpolation. Coll Redw 1998;45:1049-60.

4. Bengio Y. Learning deep architectures for AI. Found Trends Mach Learn 2009;2: $1-127$.

5. Hinton G. A practical guide to training restricted Boltzmann machines. Momentu m 2010;9:926.

6. Bengio Y. Deep learning of representations for unsupervised and transfer learning . Unsuperv Transf Learn Chall Mach Learn 2012;7:19.

7. Dedinec A, Filiposka S, Dedinec A, et al. Deep belief network based electricity 1 oad forecasting: an analysis of Macedonian case. Energy 2016;115:1688-700. 
8. O'Connor P, Neil D, Liu SC, et al. Real-time classification and sensor fusion wit h a spiking deep belief network. Front Neurosci 2013;7:178.

9. Wulsin DF, Gupta JR, Mani R, et al. Modeling EEG waveforms with semi-super vised deep belief nets: fast classification and anomaly measurement. J Neural Eng 2011;8:036015. 